\title{
Ultrasound appearance of peripheral nerves in the neck: vagus, hypoglossal and greater auricular
}

Andra Diana Curcean ${ }^{1}$, Georgeta Mihaela Rusu ${ }^{1}$, Sorin Marian Dudea ${ }^{2}$

MEDICAL IMAGING

1) Radiology Department, Academic Emergency County Hospital, ClujNapoca, Romania

2) Radiology Department, Iuliu Hatieganu University of Medicine and Pharmacy, Cluj-Napoca, Romania
DOI: $10.15386 / \mathrm{mpr}-1273$

Manuscript received: 14.01.2019

Received in revised form: 31.07.2019

Accepted: 06.09.2019

Address for correspondence:

mihageorgeta@yahoo.com

This work is licensed under a Creative Commons Attribution-NonCommercialNoDerivatives 4.0 International License

\begin{abstract}
Background and aim. Information in ultrasonography about reference values of nerves, particularly of those located in the neck is limited. The aim of the study is to demonstrate the feasibility of direct visualization of the vagus, hypoglossal and greater auricular nerves, testing the method on healthy volunteers and estimate the reference values for two perpendicular diameters and cross-sectional area (CSA).

Methods. A prospective study was carried out on 21 healthy volunteers (12 women and 9 men), recording their demographic characteristics. A $13 \mathrm{MHz}$ transducer was used on a Hitachi EUB-8500 machine. The vagus nerve was measured at the bifurcation of common carotid artery (CCA) and at the intersection of the internal jugular vein with omohyoid muscle. The hypoglossal nerve was identified as it crosses the posterolateral border of the mylohyoid muscle in the submandibular space. The auricular nerve was identified on the superficial surface of the sternocleidomastoid muscle at $1 \mathrm{~cm}$ from its posterior margin. The mean CSA on axial scans, nerve width and thickness were determined. The side-to-side and gender differences of the estimated reference values and their correlations with the weight, height, and body mass index (BMI) were evaluated.
\end{abstract}

Results. The mean ultrasound-estimated CSA was measured for the vagus nerve at CCA bifurcation $(2.14 \pm 0.79$ on the left $2.86 \pm 1.27$ on the right $)$, vagus nerve at the level of omohyoid muscle $(2.10 \pm 0.06$ on the left and $2.43 \pm 0.08$ on the right), hypoglossal nerve $(1.71 \pm 0.08$ on the left, $1.24 \pm 0.06$ on the right $)$ and greater auricular nerve $(0.90 \pm 0.53$ on the left and $0.79 \pm 0.71$ on the right $)$. The vagus nerve was significantly larger on the right side compared to the left side. Larger hypoglossal nerve was observed in men compared to women. Significant correlations were observed between weight and CSA of the greater auricular nerve.

Conclusion. Direct visualization of the vagus, greater auricular nerve and hypoglossal nerves is feasible. Reference values for the size of the studied nerves at specific anatomic landmarks were established. Side, gender and body weight differences of the nerves CSA were noted.

Keywords: ultrasonography, peripheral nerve, neck nerves, reference values

\section{Background and aims}

Over the last two decades, highresolution ultrasonography has become a reliable tool for the investigation of peripheral nerves. It provides valuable information, in many cases, not available through MRI or electrophysiological studies [1-3]. High frequency probes provide better image resolution, enhancing the morphological analysis of the nerves. As image quality increases, the cost of ultrasound decreases due to the advancements in computer and software technologies, making it an increasingly attractive method for the assessment of small, relatively superficial structures. The commonly used parameters for peripheral nerve description are crosssectional area (CSA) on axial scans, nerve width (medial to lateral diameter) and thickness (anterior to posterior diameter) $[4,5]$.

Well-defined reference values are essential requirements for high-resolution ultrasound. Although many articles describe the US appearance of nerves in various neuropathies (postoperative nerve injuries or tumors) [6-8], information 
is less abundant when it comes to normal values of peripheral nerves. Moreover, cranial nerves received less attention compared to peripheral nerves [9]. Currently, reference values for cranial nerves in healthy volunteers were reported only by a few articles [10-14].

The aim of this study is to test the feasibility of ultrasonographic visualization of the extracranial segment of cranial nerves (vagus, hypoglossal) and auricular nerve. A secondary objective is to determine the cross sectional area and two perpendicular diameters of the nerves in a normal volunteer group as a reference and to determine whether there is a statistically significant difference between average measurements for men and women, left and right side of the same volunteer and ipsilateral sides for opposite gender. The correlation between body mass index (BMI) and morphometric data for each nerve is also assessed.

\section{Methods}

\section{Demographic and clinical data}

Twenty-one healthy volunteers (12 female and 9 male, mean age $25 \pm 2$ years (range 18-28 years), mean weight $72 \pm 17 \mathrm{~kg}$ and mean height $174 \pm 10 \mathrm{~cm}$ ) participated in this study. Age, gender, handedness, height and weight were recorded, and BMI was calculated for each subject. Exclusion criteria were history of nerve injury, neck surgery and the presence of scars which could influence the examination.

High-resolution ultrasound examination of the nerves

The selected subjects were examined by a sonographer (D.S.) with over 30 years of experience, using a Hitachi EUB-8500 ultrasound machine (Hitachi Medical Corporation, Tokyo, Japan) with a 5-13 MHz linear probe. Participants were placed in supine position with slight head extension and contralateral rotation to the scanned nerve. Individually optimized settings were used for each volunteer with respect to gain, depth and focus. An overview scan in the neck region was performed at first in order to identify the vagus nerve and its course, holding the transducer in axial position. The aim was to find a round, hypoechoic structure in axial view, surrounded by a slightly hyperechoic border which corresponds to the normal structure of the nerve. The vagus nerve was located in the carotid sheath, antero-laterally to the common carotid artery and dorsally to the internal jugular vein as it was described in former studies [15,16]. Individual fascicles were visible in the nerve, giving the structure a "honeycomb" appearance with two to five hypoechoic oval fascicles separated by hyperechoic epineurial tissue. Color Doppler was used, when necessary, to avoid misinterpreting a small vessel as a nerve.

Ultrasound was used to scan the cervical segment of the nerve at two predetermined sites: the proximal site was at the level of the carotid sinus just before the common carotid artery bifurcation (Figure 1) and the distal site at the intersection between omohyoid muscle and internal jugular vein (Figure 2). Minimal pressure was applied during the ultrasound examination in order to prevent nerve compression, keeping the transducer perpendicular to the nerve axis. Anatomical variations of the nerves were noted.

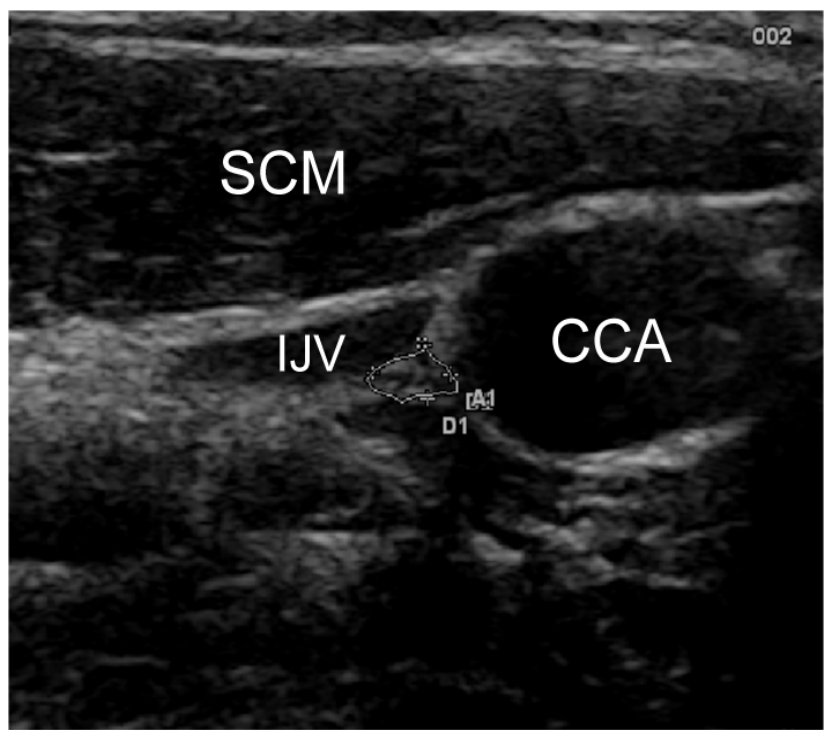

Figure 1. Vagus nerve at the common carotid artery bifurcation. $\mathrm{SCM}=$ sternocleidomastoid muscle, IJV = internal jugular vein, $\mathrm{CCA}=$ common carotid artery.



Figure 2. Vagus nerve at the intersection between omohyoid muscle and internal jugular vein. OMO = omohyoid muscle, IJV $=$ internal jugular vein, $\mathrm{CCA}=$ common carotid artery. 
Next, the probe was moved cranially, to the submandibular region where the hypoglossal nerve was identified adjacent to the intermediate tendon of the digastric muscle, beneath the surface of the mylohyoid muscle and next to the lingual nerve (Figure 3).

This is a modification of the technique described by Meng et al. [14] in which the posterolateral border of the mylohyoid muscle was used as a reference.

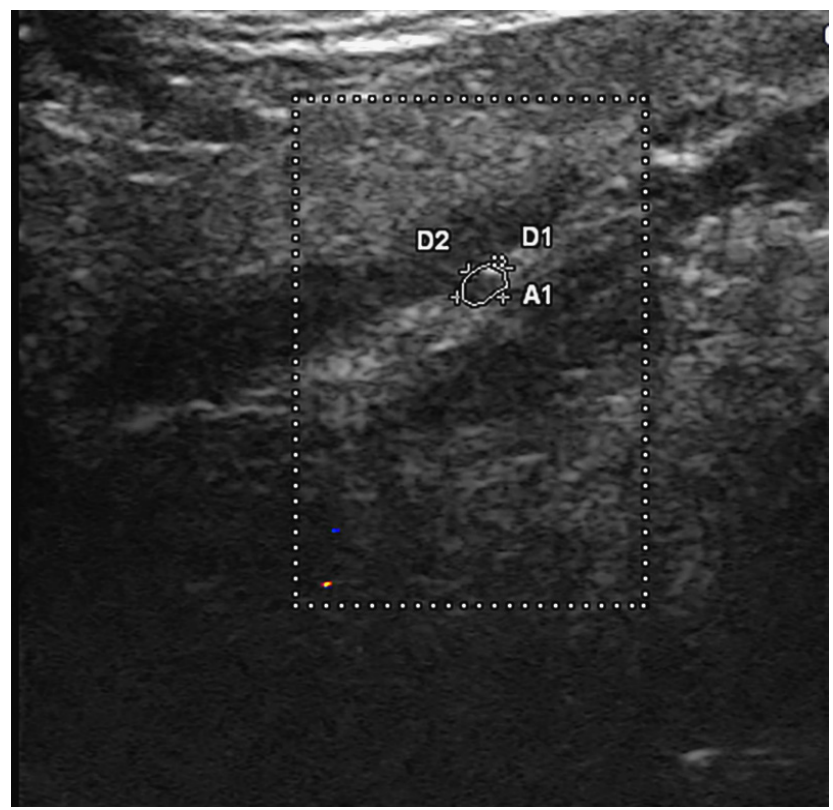

Figure 3. Hypoglossal nerve. Color Doppler was used to demonstrate that the structure is not a small vessel.

The greater auricular nerve was identified on the external surface of the sternocleidomastoid (SCM) muscle, as it runs around its posterior border and then following a cranial course towards the ear. The nerve was followed superficially, underneath the platysma muscle, and measured at about $1 \mathrm{~cm}$ from the external border of the sternocleidomastoid muscle (Figure 4).

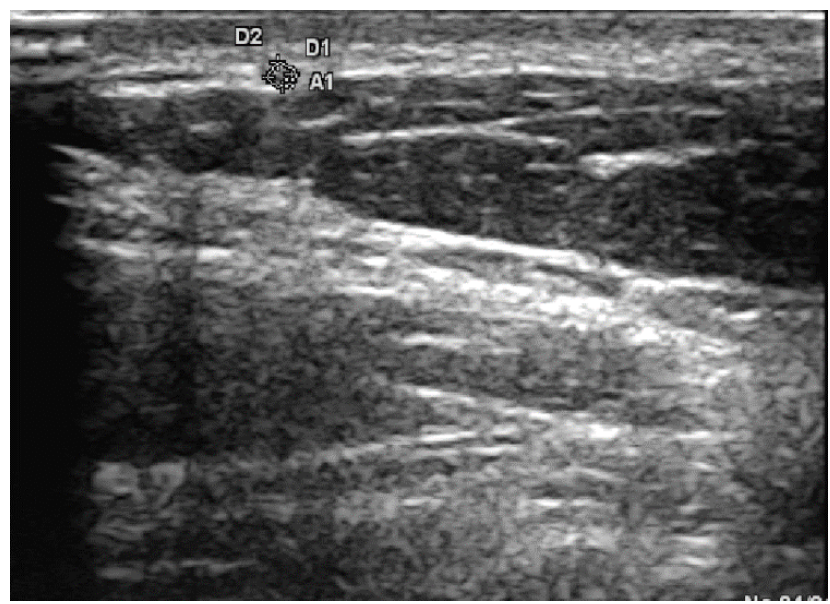

Figure 4. The greater aurricular nerve on the surface of sternocleidomastoid muscle.
Cross-sectional areas, antero-posterior and transverse diameters were recorded for each nerve. The trace function was used to measure the cross-sectional area along the inner border of the hyperechoic rim. Images including measurements of the analyzed nerves were recorded. Data was organized into a spreadsheet.

\section{Statistical analysis}

As the machine could not accurately measure areas below $0.01 \mathrm{~cm}^{2}$, a value of $0.005 \mathrm{~cm}^{2}$ was conventionally attributed to all CSAs in this category.

Statistical analyses were performed using MedCalc Statistical Software, version 17.5.5 (Medcalc Software bvba, Ostend, Belgium). Normal distribution of nerve measurements was demonstrated using Shapiro-Wilk test. Student paired t-test was applied for side-to-side and gender comparisons for continuous and normally distributed variables. The threshold of significance was set at $p$-value $=0.05$. Spearman's rho correlation coefficient was used to correlate nerve measurements with height, weight and body mass index. Similarly, the p-value $<0.05$ was considered statistically significant.

The study was approved by the Scientific Research Ethics Committee of the University.

\section{Results}

The study included 21 subjects, the basic demographic data being presented in Table I. The nerve identification rate using ultrasound is shown in Table II.

Table I. Demographic data of the volunteer group.

$\begin{array}{lccc}\text { Parameters } & \text { Mean + SD } & \text { Male } & \text { Female } \\ \text { Age (years) } & 25 \pm 2 & 24.5 \pm 2.96 & 25.5 \pm 0.9 \\ \text { Weight (kg) } & 72 \pm 17 & 84.2 \pm 11.3 & 64.5 \pm 16 \\ \text { Height (cm) } & 174 \pm 10 & 183 \pm 5.54 & 168 \pm 7.33 \\ \text { Body Mass Index } & 23.6 \pm 4.42 & 24.9 \pm 3.78 & 22.63 \pm 4.76\end{array}$

$\mathrm{SD}=$ standard deviation

Table II. Nerve identification rates using ultrasound.

\begin{tabular}{l|c|c|c|}
\multirow{2}{*}{ Nerve } & \multirow{2}{*}{ Side } & \multicolumn{2}{|c}{ Ultrasound identification rate } \\
\cline { 3 - 4 } & & No & $\%$ \\
\hline \multirow{2}{*}{ Vagus proximal } & Left & $21 / 21$ & 100 \\
Vagus distal & Right & $21 / 21$ & 100 \\
\multirow{2}{*}{ Hypoglossal } & Left & $20 / 21$ & 95 \\
\multirow{2}{*}{ Greater auricular } & Right & $21 / 21$ & 100 \\
& Left & $17 / 21$ & 80 \\
& Right & $21 / 21$ & 100 \\
& Right & $20 / 21$ & 95 \\
& & $20 / 21$ & 95
\end{tabular}

The measurement results are summarized in Table III. Side to side and male to female comparisons were made for each measurement.

The results of the "Shapiro-Wilks" test was illustrated for three nerve sites in figures 5-7, following a normal distribution. 
Table III. Ultrasound scan nerve measurements in healthy volunteers.

\begin{tabular}{|c|c|c|c|c|c|c|}
\hline Side & $\mathbf{A P}(\mathbf{m m})$ & $\begin{array}{l}\text { Left side } \\
\text { TR(mm) }\end{array}$ & $\mathrm{CSA}\left(\mathrm{mm}^{2}\right)$ & $\mathbf{A P}(\mathbf{m m})$ & $\begin{array}{c}\text { Right side } \\
\text { TR(mm) }\end{array}$ & $\mathrm{CSA}\left(\mathrm{mm}^{2}\right)$ \\
\hline Vagus - proximal & $1.73 \pm 0.31$ & $1.76 \pm 0.40$ & $2.10 \pm 0.06$ & $1.84 \pm 0.30$ & $2.03 \pm 0.40$ & $2.43 \pm 0.08$ \\
\hline Vagus - distal & $1.73 \pm 0.43$ & $1.79 \pm 0.39$ & $2.14 \pm 0.79$ & $1.91 \pm 0.42$ & $2.05 \pm 0.47$ & $2.86 \pm 1.27$ \\
\hline Hypoglossal & $1.51 \pm 0.62$ & $1.60 \pm 0.38$ & $1.71 \pm 0.08$ & $1.46 \pm 0.38$ & $1.46 \pm 0.40$ & $1.24 \pm 0.06$ \\
\hline Greater auricular & $1.11 \pm 0.39$ & $1.06 \pm 0.50$ & $0.90 \pm 0.53$ & $0.99 \pm 0.43$ & $0.79 \pm 0.71$ & $0.75 \pm 0.21$ \\
\hline $\begin{array}{ll}\text { Nerve } & \text { Gender } \\
\end{array}$ & $\mathbf{A P}(\mathbf{m m})$ & $\begin{array}{c}\text { Male } \\
\text { TR(mm) }\end{array}$ & $\mathrm{CSA}\left(\mathrm{mm}^{2}\right)$ & $\mathbf{A P}(\mathbf{m m})$ & $\begin{array}{l}\text { Female } \\
\text { TR(mm) }\end{array}$ & $\operatorname{CSA}\left(\mathrm{mm}^{2}\right)$ \\
\hline Vagus - proximal & $1.69 \pm 0.36$ & $1.97 \pm 0.48$ & $2.57 \pm 1.16$ & $1.85 \pm 0.27$ & $1.88 \pm 0.39$ & $2.33 \pm 1.16$ \\
\hline Vagus - distal & $1.91 \pm 0.41$ & $2.02 \pm 0.46$ & $2.7 \pm 1.12$ & $1.77 \pm 0.46$ & $1.86 \pm 0.44$ & $2.34 \pm 0.71$ \\
\hline Hypoglossal & $1.55 \pm 0.66$ & $1.64 \pm 0.39$ & $1.66 \pm 0.84$ & $1.42 \pm 0.27$ & $1.45 \pm 0.42$ & $1.4 \pm 0.68$ \\
\hline Greater auricular & $1.24 \pm 0.47$ & $1.00 \pm 0.37$ & $1.05 \pm 0.59$ & $0.95 \pm 0.38$ & $1.05 \pm 0.55$ & $0.92 \pm 0.32$ \\
\hline
\end{tabular}

$\mathrm{AP}=$ antero-posterior diameter; $\mathrm{TR}=$ transverse diameter; $\mathrm{CSA}=$ cross-sectional area; Vagus proximal $=$ at the carotid sinus; Vagus distal $=$ at intersection between omohyoid muscle and internal jugular vein .

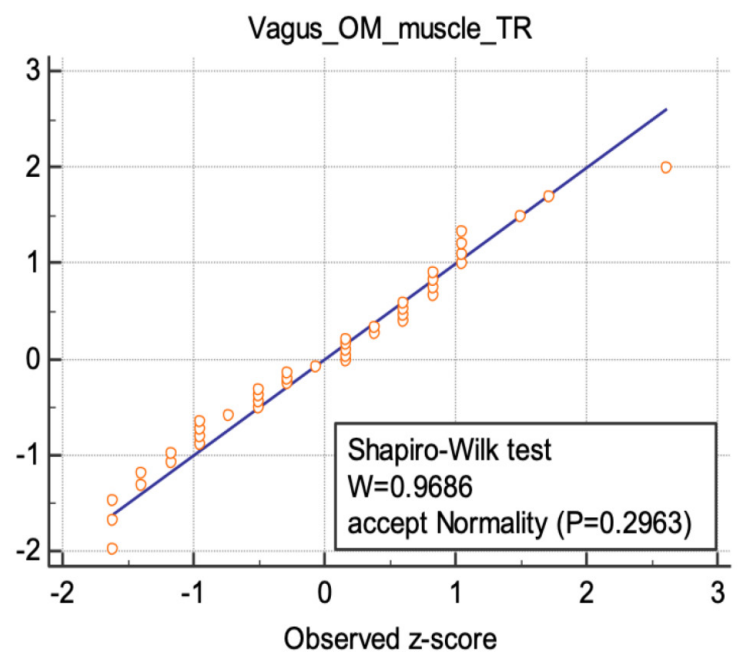

Figure 5. Normal distribution illustrated for the transverse diameter values of vagus nerve at the intersection between omohyoid muscle and internal jugular vein.

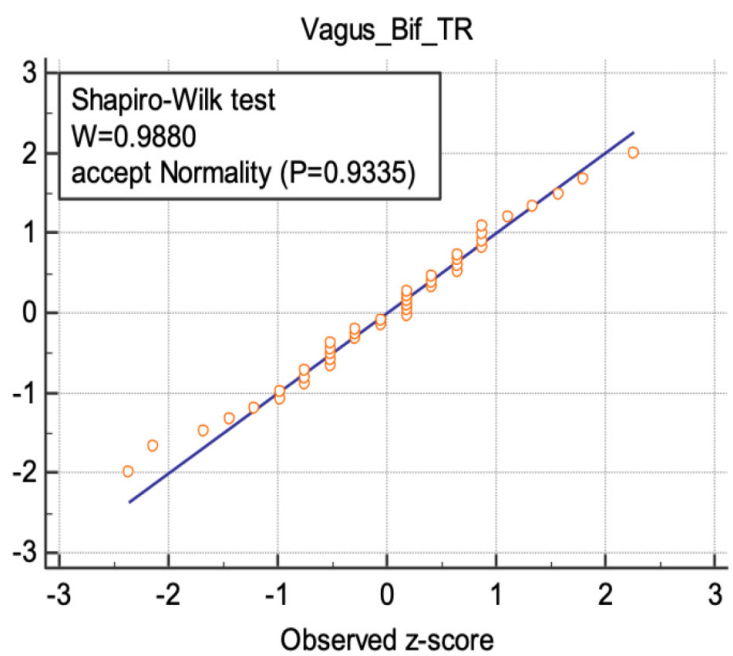

Figure 6. Normal distribution illustrated for the transverse diameter values of vagus nerve at the common carotid artery bifurcation.

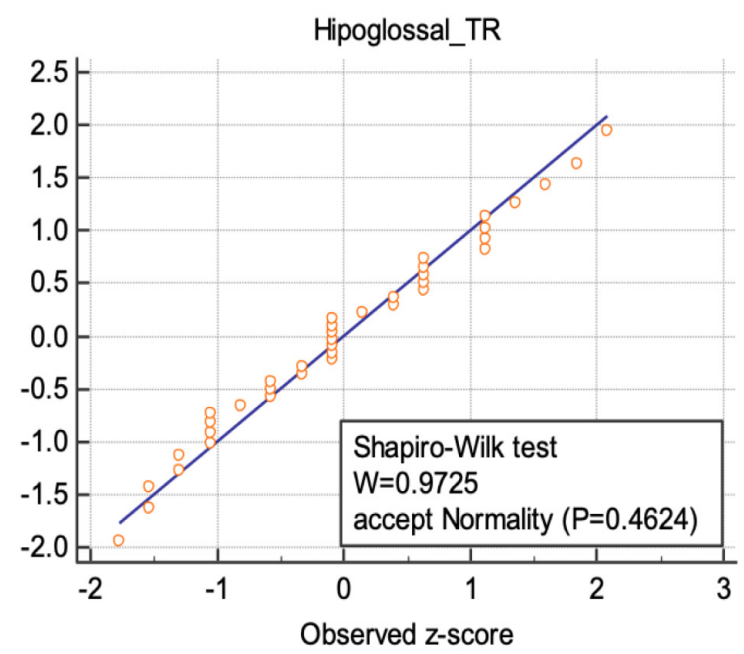

Figure 7. Normal distribution illustrated for the transverse diameter values of hypoglossal nerve. 
The vagus nerve cross-sectional area showed significant differences between opposite sides, the right side being larger than the left side at the intersection between the internal jugular vein and omohyoid muscle $(\mathrm{p}=0.035)$. Likewise, at the carotid sinus site, the transverse diameter of the right vagus nerve was significantly larger than the contralateral one $(p=0.039)$. There was no significant difference between male and female in vagus nerve measurements or between different genders or opposite sides in greater auricular or hypoglossal nerve. The gender difference regarding hypoglossal nerve cross sectional area $\left(1 \pm 0.4 \mathrm{~cm}^{2}\right.$ in women and $1.5 \pm 0.7 \mathrm{~cm}^{2}$ in men $)$ was not statistically significant. Of all measurements, only the greater auricular nerve correlated with weight (Figure 8). The age correlation was not assessed as the volunteers were in the range of 18-28 years.

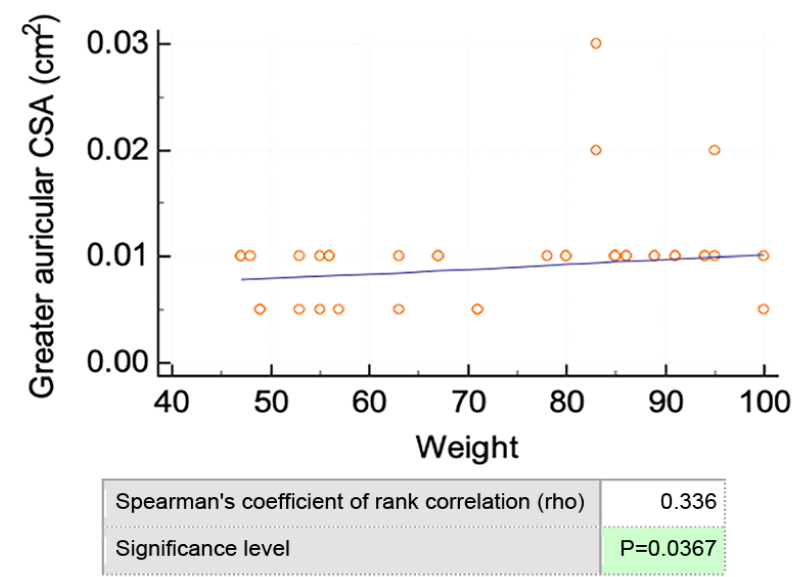

Figure 8. Correlation between greater auricular CSA and weight.

\section{Anatomical variants}

In most cases, the vagus nerve was located posteriorly between the jugular vein and internal carotid artery. In one case, two vagus nerve stalks were identified on the right side lying between the neck vessels and converging caudally in the carotid sheath (Figure 9). On the opposite side, a single vagus nerve was visualized following the usual course.

In another subject, the omohyoid muscle separated the common carotid artery from the jugular vein, passing between the two (Figure 10). The vagus nerve was located superficially, on the medial side of the jugular vein and anteriorly to the omohyoid muscle.

No anatomical variation regarding the position or number of the greater auricular or the hypoglossal nerves were observed.

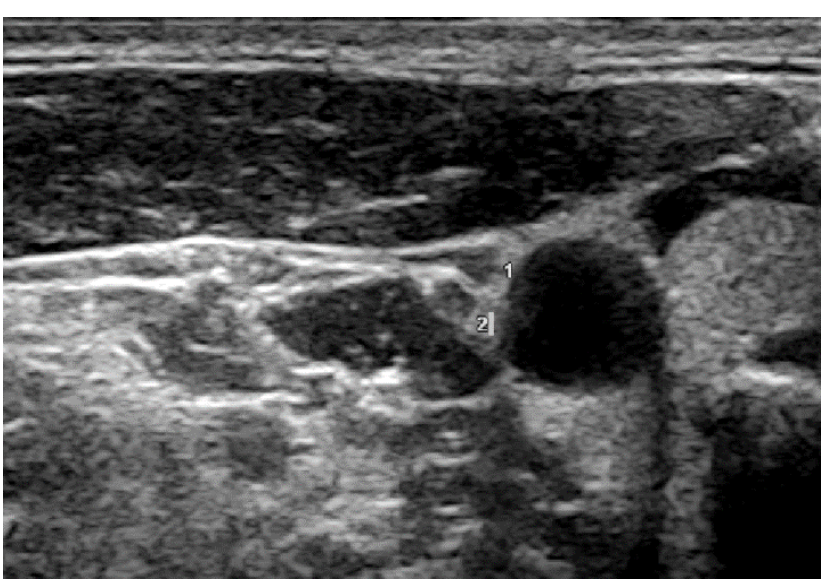

Figure 9. Anatomical variation of the vagus nerve. Two vagus nerve stalks on the right side lying between the neck vessels.



Figure 10. Anatomical variation of the vagus nerve located on the surface of omohyoid muscle alongside the jugular vein. SCM = sternocleidomastoid muscle, IJV = internal jugular vein, $\mathrm{CCA}=$ common carotid artery, $\mathrm{OMO}=$ omohyoid muscle.

\section{Discussion}

The present study demonstrated the feasibility of ultrasound identification of cervical nerves (vagus, auricular and hypoglossal) at well-defined anatomical landmarks. The vagus nerve was clearly visible in all volunteers despite having the described variations in its course. The somehow limited visualization of the greater auricular and hypoglossal nerve may be attributed to small size, ultrasound frequency limitation or anatomic variants.

Previous studies focused on cervical nerves localization, anatomical variations and their branches (the recurrent laryngeal or the superior laryngeal nerve) in order to avoid iatrogenic injury during neck surgery or to assess postoperative nerve function [10,17-21]. Some studies reported size variation of the nerves in pathological conditions $[12,22]$, but only a few focused on generating reference values in normal subjects $[1,5,23]$. The intent of the current study is to assess a standardized approach of some cervical nerves by means of ultrasound. 


\section{The vagus nerve}

The vagus nerve was identified in the posterior triangular space formed between the common carotid artery and jugular vein in $90 \%$ of cases. Anatomical variants $(10 \%)$ included two vagus nerve stalks on one side and a medial position in relation to the jugular vein, both crossing the superficial side of the omohyoid muscle, to the best of our knowledge not previously described. However, an anterior-medial position and a posterior-lateral one in relation to the common carotid artery were previously noted [10]. This emphasizes the complex anatomical variations in the vagal nerve course that need to be further assessed. This particular knowledge may be of interest in assessing nerve pathology or prior to neck surgery (when the course of a nerve is different from its expected route).

Cross-sectional values of the vagus nerve in the current study nerve were similar to the ones reported in a recent study [10] as illustrated in Table IV.

There are noteworthy discrepancies in literature, Cartwright et al. [1] and Tawfik et al. [12] reporting significantly larger CSA for vagus nerve compared to Peltz et al. [10] and Grimm et al. [17], who described values three times smaller. The latter are comparable with the current study. Different measurements were reported despite tracing the nerve contour in a similar manner (inside the hyperechoic rim). These different results in CSA were explained by a different study cohort which included non-Caucasians and a more heterogeneous study sample [10]. Another important aspect is that image quality does not depend solely on transducer frequency but also on software algorithms that reduce artifacts, noise or speckle. Reducing motion artifacts might be of special importance when it comes to assessing the vagus nerve which is placed next to the pulsating common carotid artery. Moreover, different imaging tasks (analyzing a normal or an altered nerve structure), or patients (short necked or obese), may require different ultrasound settings [24]. Former studies marked only the cross-sectional areas, this study being the first to measure the anterior-posterior and transversal diameters. These measurements could be useful when using lower frequency probes as the nerve contour might not be clearly visible and therefore not easily traceable.

A significant difference between the right and the left vagus nerve cross-sectional area was noticed. This finding is validated by a previous study which clearly demonstrated this side-dependent difference by replicating the results using measurements from two different ultrasound systems and comparing online as well as offline-measurements
[10]. Similarly, the transversal diameter of the right vagus nerve was significantly larger than the contralateral one in the current study. These results were not replicated by other studies on peripheral nerves so this issue needs to be further addressed. It is known that the vagus nerve provides an asymmetric innervation for the organs, therefore it is likely to have a different distribution of nerve fibers in opposite sides [15]. No side-to-side differences were found in the single anatomical study comparing the left and the right vagus nerve in the neck region [15]. Even though an increased CSA of peripheral nerves on the dominant hand side was not demonstrated before [1], we noticed that 2 out of 3 left handed subjects had a larger vagus nerve on the left side compared to the non-dominant side. In order to test the plausibility of this observation, this side-dependent difference in relation to the dominant hand should be further analyzed in larger groups of volunteers.

Similar to previous studies $[1,10,12,22]$, no significant correlation between nerve measurements and demographic data were found. However, a negative correlation with age was reported in a recent study on vagus nerve and also described in other articles analyzing peripheral nerves $[10,11]$. These age-dependent changes in nerve size are thought to be caused by age-dependent axonal degeneration. The former study also divided their volunteers in two subgroups ( $\leq 50$ years and $>50$ years) and found that the CSA in the younger subgroup was larger than the one in the older subgroup, supporting the age-dependent degeneration hypothesis. This demonstrates the need for age-specific reference values. Their exclusion criteria in the older subgroup did not include diabetes which was proved to reduce vagus nerve sizes in a previous study [12]. As most of the volunteers in the current study were 25 years old, the correlation between nerve measurements and age was not assessed.

A standardized, methodological approach in nerve assessment requires reliable anatomical landmarks. These topographical guides allow an easy and reproducible identification of a nerve at various sites [24]. Similar to former studies, we used the carotid bifurcation as a proximal reference point to measure the cross-sectional area of the vagus nerve $[1,10]$. Thyroid gland, sternocleidomastoid muscle or thyroid cartilage were also used as guides for nerve assessment. In the current study, the distal site of the vagus nerve was measured at the intersection between the omohyoid muscle and the internal jugular vein, a more specific and easily identifiable landmark not used before, to the best of our knowledge.

Table IV. Vagus nerve measurements.

\begin{tabular}{|c|c|c|c|c|}
\hline & \multicolumn{2}{|c|}{ Right vagus nerve } & \multicolumn{2}{|c|}{ Left vagus nerve } \\
\hline & proximal & distal & proximal & distal \\
\hline Current study & $2.43 \pm 0.08 \mathrm{~cm}^{2}$ & $2.86 \pm 1.27 \mathrm{~cm}^{2}$ & $2.1 \pm 0.06 \mathrm{~cm}^{2}$ & $2.14 \pm 0.79 \mathrm{~cm}^{2}$ \\
\hline Peltz et al. [10] & $2.7 \pm 0.6 \mathrm{~cm}^{2}$ & $2.6 \pm 0.6 \mathrm{~cm}^{2}$ & $2.1 \pm 0.5 \mathrm{~cm}^{2}$ & $1.9 \pm 0.4 \mathrm{~cm}^{2}$ \\
\hline
\end{tabular}




\section{The hypoglossal nerve}

The hypoglossal nerve was visualized in all volunteers on the right side $(21 / 21)$ and in $80 \%$ of cases on the left side (17/21). The scanning difficulties included patients with short necks and beards which decreased the image quality and made the nerve measurements more challenging. History of neck surgery or radiation therapy may also impede ultrasound assessment, but this was not the case in the current study.

Magnetic resonance is described as being the best imaging method for assessing the extracranial segment of the hypoglossal nerve, but it is limited by small nerve sizes [25]. Nevertheless, one study proposed an imaging protocol for the nerve visualization using ultrasound applied on healthy volunteers and patients [14]. The correct identification of the nerve was also confirmed, in the same study, by ultrasound guided ink injections and dissection of cadaveric specimens. The hypoglossal nerve was successfully identified between the mylohyoid and hyoglossal muscles, this anatomical landmark being comparable to the one used in this study. The crosssectional area measured at the posterolateral rim of the mylohyoid muscle $\left(1.9 \pm 0.6 \mathrm{~mm}^{2}\right.$ on both sides $)$ is similar to the present study $(1.71 \pm 0.08$ on the left side and $1.24 \pm$ 0.06 on the right side). To our knowledge, only one article provided reference values for the hypoglossal nerve, thus this may be a starting point for further studies using highresolution ultrasound to analyze the cranial nerves.

\section{The greater auricular nerve}

Available data regarding the normal and pathological appearance of the greater auricular nerve is limited [26,27]. The nerve was identified in 20/21 of cases on both sides on the superficial side of the sternocleidomastoid muscle, despite its small size. This anatomical landmark is reliable, as the nerve was ink marked and successfully identified by dissection in a previous study [13]. Its superficial course makes the identification much easier compared to other nerves in the region. Only one study confirms the feasibility of nerve identification and reports reference values for the greater auricular nerve in 10 healthy volunteers while also describing a few pathological aspects of the structure. Identifying and measuring the greater auricular nerve may be of interest in facial nerve reconstruction after parotidectomy [28].

A previous study reported a mean diameter of 1.4 $\mathrm{mm}$, a larger diameter than in the present study which includes two perpendicular measurements (1.11/1.06 $\mathrm{mm}$ on the left and $0.99 / 0.79 \mathrm{~mm}$ on the right) [13]. This discrepancy in size can be explained by different measurement sites. Formerly, the nerve was measured on the deep surface of the sternocleidomastoid muscle whereas in the present study the diameter was assessed after the nerve turns cranially, crossing the posterior border of the muscle, therefore after the point of bifurcation into the anterior and posterior branch.
The high standard deviation suggests significant differences in nerve diameter which could be explained by the inter-individual variation of the bifurcation point that occurs before or after the chosen scanning point.

The limitations of this study included a relatively small number of volunteers. Lack of information on intraand inter-observer reliability is also a drawback. A previous study showed a good intra- and inter-rater agreement in cross sectional areas for the vagus nerve. Due to a good agreement between offline- and online-measurements, dedicated software for nerve measurements in not required [10]. Another drawback of this study is the lack of age diversity in volunteers who were around 25 years old. Therefore, these data can be used to define reference values only in younger adults, as it was previously suggested that nerve size may vary with age [10].

Motion artefacts caused by common carotid artery pulsations could have also influenced the current study. Electrocardiogram gating could be useful in overcoming this impediment. In addition, in order to measure the smallest CSA, the plane of image acquisition must be perpendicular on the nerve course. Even slight deflection off the axial plane may induce significant increase in nerve area, thus overestimating the ultrasound measurements. This might be the cause of measurement discrepancies between different studies. The use of 3D ultrasound could be the answer for both problems, as it can facilitate the smallest CSA measurement of the nerve and also visualize the deflection of the nearby artery wall by pulse wave [29].

In conclusion, ultrasound is a highly feasible method in peripheral nerve assessment. Anatomical landmarks facilitate nerve identification. Side differences should be taken into consideration while assessing the vagus nerve as multiple studies showed a larger cross-sectional area on the right. Further data is required in order to establish specific imaging protocols, to generate reference values and to correlate nerve measurements with demographic data.

\section{References}

1. Cartwright MS, Passmore LV, Yoon JS, Brown ME, Caress JB, Walker FO. Cross-sectional area reference values for nerve ultrasonography. Muscle Nerve. 2008;37:566-571.

2. Cartwright MS, Demar S, Griffin LP, Balakrishnan N, Harris JM, Walker FO. Validity and reliability of nerve and muscle ultrasound. Muscle Nerve. 2013;47:515-521.

3. Tawfik EA, Walker FO, Cartwright MS. Neuromuscular ultrasound of cranial nerves. J Clin Neurol. 2015;11:109-121.

4. Walker FO. Ultrasonography in Peripheral Nervous System Diagnosis. Continuum (Minneap Minn). 2017;23(5, Peripheral Nerve and Motor Neuron Disorders):1276-1294.

5. Boehm J, Scheidl E, Bereczki D, Schelle T, Arányi Z. Highresolution ultrasonography of peripheral nerves: measurements on 14 nerve segments in 56 healthy subjects and reliability assessments. Ultraschall Med. 2014;35:459-467. 
6. Peer S, Bodner G, Meirer R, Willeit J, Piza-Katzer H. Examination of postoperative peripheral nerve lesions with high-resolution sonography. AJR Am J Roentgenol. 2001; 177:415-419.

7. Bhatt S, Verma P, Meena N, Tandon A, Tanveer N, Bansal D. Glomus vagale tumour, can it be diagnosed only on sonography? J Ultrasound. 2017;20:73-79.

8. Liu B, Zhang Y, Zhang L, Zhang F, Li H, Li S, et al. A rare case of bilateral cervical vagal neurofibromas: role of highresolution ultrasound. BMC Neurol. 2017;17:26.

9. Kerasnoudis A, Pitarokoili K, Behrendt V, Gold R, Yoon MS. Cross sectional area reference values for sonography of peripheral nerves and brachial plexus. Clin Neurophysiol. 2013;124:1881-1888.

10. Pelz JO, Belau E, Henn P, Hammer N, Classen J, Weise D. Sonographic evaluation of the vagus nerves: Protocol, reference values, and side-to-side differences. Muscle Nerve. 2018;57:766-771.

11. Qrimli M, Ebadi H, Breiner A, Siddiqui H, Alabdali M, Abraham A, et al. Reference values for ultrasonograpy of peripheral nerves. Muscle Nerve. 2016;53:538-544.

12. Tawfik EA, Walker FO, Cartwright MS, El-Hilaly RA. Diagnostic Ultrasound of the Vagus Nerve in Patients with Diabetes. J Neuroimaging. 2017;27:589-593.

13. Lieba-Samal D, Pivec C, Platzgummer H, Gruber GM, Seidel S, Bernathova M, et al. High-Resolution Ultrasound for Diagnostic Assessment of the Great Auricular Nerve-Normal and First Pathologic Findings. Ultraschall Med. 2015;36:342-347.

14. Meng S, Reissig LF, Tzou CH, Meng K, Grisold W, Weninger W. Ultrasound of the Hypoglossal Nerve in the Neck: Visualization and Initial Clinical Experience with Patients. AJNR Am J Neuroradiol. 2016;37:354-359.

15. Al-Kureischi K. Topographic anatomy and fiber diameter of the human vagal trunk. Acta Anat (Basel). 1979;103:252258.

16. Lee JH, Cheng KL, Choi YJ, Baek JH. High-resolution Imaging of Neural Anatomy and Pathology of the Neck. Korean J Radiol. 2017;18:180-193.

17. Grimm A, Décard BF, Axer H. Ultrasonography of the peripheral nervous system in the early stage of GuillainBarré syndrome. J Peripher Nerv Syst. 2014;19:234-241.

18. Kaur B, Tang R, Sawka A, Krebs C, Vaghadia H. A method for ultrasonographic visualization and injection of the superior laryngeal nerve: volunteer study and cadaver simulation. Anesth Analg. 2012;115:1242-1245.

19. Kundra P, Kumar K, Allampalli V, Anathkrishnan R, Gopalakrishnan S, Elangovan S. Use of ultrasound to assess superior and recurrent laryngeal nerve function immediately after thyroid surgery. Anaesthesia. 2012;67:301-302.

20. Snosek M, Margarit G, Burns D, Hage R, Tubbs S LM. Localization of the recurrent laryngeal nerve using ultrasound. The Federation of American Societies for Experimental Biology Journal. 2014;28:923-925.

21. Park JK, Jeong SY, Lee JH, Lim GC, Chang JW. Variations in the course of the cervical vagus nerve on thyroid ultrasonography. AJNR Am J Neuroradiol. 2011;32:1178-1181.

22. Tawfik EA, Walker FO, Cartwright MS. A Pilot Study of Diagnostic Neuromuscular Ultrasound in Bell's Palsy. J Neuroimaging. 2015;25:564-570.

23. Cartwright MS, Mayans DR, Gillson NA, Griffin LP, Walker FO. Nerve cross-sectional area in extremes of age. Muscle Nerve. 2013;47:890-893.

24. Spiss V, Peer S, Judmaier W, Brenner E. Nerves in the Neck. In: Peer S, Gruber H. (eds.) Atlas of peripheral nerve ultrasound: with anatomic and MRI correlation. Heidelberg, Germany: Springer; 2013. pp. 29-42.

25. Alves P. Imaging the hypoglossal nerve. Eur J Radiol. 2010;74:368-377.

26. Thallaj A, Marhofer P, Moriggl B, Delvi BM, Kettner SC, Almajed M. Great auricular nerve blockade using high resolution ultrasound: a volunteer study. Anaesthesia. 2010;65:836-840.

27. Jeon Y, Kim S. Treatment of great auricular neuralgia with real-time ultrasound-guided great auricular nerve block: A case report and review of the literature. Medicine (Baltimore). 2017;96:e6325.

28. Bahadir O, Livaoglu M, Ural A. Adenoid cystic carcinoma of the parotid gland: Anastamosis of the facial nerve with the great auricular nerve after radical parotidectomy. Indian J Plast Surg. 2008;41:201-205.

29. Macharzina RR, Kocher S, Messé SR, Rutkowski T, Hoffmann F, Vogt M, et al. 4-Dimensionally Guided 3-Dimensional Color-Doppler Ultrasonography Quantifies Carotid Artery Stenosis With High Reproducibility and Accuracy. JACC Cardiovasc Imaging. 2018;11:386-396. 